\title{
CONTENT OF FATHER AND MOTHER STEREOTYPES IN JAPAN, COMPARED TO OVERALL GENDER STEREOTYPES
}

\author{
Mizuka Ohtaka \\ Department of Politics and Public Administration, Faculty of Law, Yamanashi Gakuin University (Japan)
}

\begin{abstract}
Suzuki (2017) reviewed the studies on gender inequality and concluded that gender stereotypes contribute to the persistence of gender discrimination in the workplace and at home. It has also been verified that the content of father (Troilo, 2013) and mother stereotypes (Ganong \& Coleman, 1995) differed from overall gender stereotypes in American society. This study investigated whether the content of father and mother stereotypes was dissimilar to that of overall gender stereotypes in Japan. That is, does the content of father (versus men) and mother (versus women) stereotypes differ from the typically held gender stereotypes? A survey was conducted among undergraduates $(N=266$; Men $=106$, Women $=160)$, with a mean age of 19.05 years $(S D=1.02$ years). The results indicated that the content of father stereotype was different from that of men and that of mother stereotype was different from that of women. This implies that the idea that 'fathers (rather than men) should work outside the home and mothers (rather than women) should keep the house', is held in Japanese society. In the future, it would be helpful to examine not only explicit stereotypes but also implicit stereotypes about fathers and mothers. Further, it would be useful to study stereotypes held by older and/or less educated adults.
\end{abstract}

Keywords: Stereotype, father, mother, gender.

\section{Introduction}

Gender stereotype is identified as one of the factors contributing to gender inequality in offices and families (Suzuki, 2017, for review). Troilo (2013) demonstrated that in the U.S.A., the content of the father stereotype was dissimilar to that for men (e.g., American fathers were thought to be Hardworking and Busy, when compared with American men). Similarly, Ganong and Coleman (1995) substantiated that the content of the mother stereotype differed from that for women (e.g., American mothers were believed to Act for the good of family and Favor her own children, when compared with American women). In Japan too, are parent stereotypes different from overall gender stereotypes? This study tries to verify whether in Japan, the content of father stereotype differs from that for men and whether the content of mother stereotype differs from that for women.

\section{Methods}

This study conducted a survey among 266 undergraduates. They were 18-22 years old, of both genders, unmarried, born and raised in Japan, and their native language was Japanese. They randomly received one of four self-report questionnaires on either fathers, mothers, men, or women, followed by the 85 descriptors (Table 1). These descriptors were generated from two pilot studies in a similar way to previous studies (Ganong \& Coleman, 1995; Troilo, 2013).

They were asked to list a percentage for each descriptor on a 11-point scale ranging from 0 to $100 \%$ (e.g., 'What percentage of Japanese fathers do you think have each of the following descriptors? For example, if you think that all Japanese fathers have the characteristic, you would choose $100 \%$. If you think that $20 \%$ of Japanese fathers have the characteristic, you would choose $20 \%$. Do not think of the fathers you know, but of fathers in general'). The 85 descriptors were randomly sorted to reduce order effects. 


\section{Results}

\subsection{Respondents}

Table 1. The Results of Analysis of Variance.

\begin{tabular}{|c|c|c|c|c|c|c|c|c|c|c|}
\hline Category & Descriptors & Fathers & Mothers & Men & Women & & & parent & sex & interaction \\
\hline & Strong & 63.24 & 49.86 & 68.28 & 31.45 & $F(3,258)=49.62$ & *** & $* *$ & $* * *$ & $* * *$ \\
\hline Men $>$ & Powerful & 64.14 & 52.39 & 65.34 & 36.45 & $F(3,257)=32.26$ & $* * *$ & ** & *** & $* * *$ \\
\hline \multirow[t]{2}{*}{ Fathers } & Lazy & 54.29 & 47.89 & 62.20 & 50.00 & $F(3,257)=8.01$ & $* * *$ & $*$ & *** & \\
\hline & Selfish & 50.14 & 40.14 & 55.00 & 47.90 & $F(3,256)=7.52$ & $* * *$ & $* *$ & $* * *$ & \\
\hline & Physically strong & 61.86 & 48.03 & 68.47 & 36.07 & $F(3,257)=34.25$ & *** & & **** & $* * *$ \\
\hline $\begin{array}{l}\text { Men }= \\
\text { Fatherc }\end{array}$ & Strong constitution & 62.06 & 50.70 & 64.92 & 40.48 & $F(3,256)=19.90$ & $* * *$ & & $* * *$ & ** \\
\hline & Work than children & 52.54 & 33.19 & 56.27 & 38.23 & $F(3,257)=22.17$ & $* * *$ & $\begin{array}{c}t \\
\end{array}$ & $* * *$ & \\
\hline & Work & 85.51 & 64.20 & 79.65 & 58.39 & $F(3,253)=35.25$ & *** & ** & **** & \\
\hline & Hardworking & 76.96 & 65.29 & 70.18 & 60.97 & $F(3,252)=10.00$ & $* * *$ & $*$ & $* * *$ & \\
\hline & Breadwinner & 68.57 & 49.43 & 60.18 & 28.23 & $F(3,255)=40.57$ & $* * *$ & $* * *$ & $* * *$ & $*$ \\
\hline $\begin{array}{l}\text { Mien } \\
\text { Fathers }\end{array}$ & Big & 62.86 & 44.71 & 62.54 & 32.10 & $F(3,255)=40.31$ & *** & $* *$ & *** & ** \\
\hline & Masculine & 62.43 & 45.74 & 56.55 & 33.87 & $F(3,254)=24.74$ & *** & ** & *** & \\
\hline & Looking down on & 57.25 & 55.44 & 56.72 & 42.62 & $F(3,252)=7.70$ & $* * *$ & $* *$ & $* *$ & * \\
\hline & Well built & 56.86 & 45.00 & 55.42 & 32.26 & $F(3,257)=25.65$ & $* * *$ & $* *$ & $* * *$ & $*$ \\
\hline & Busy & 74.43 & 75.44 & 66.03 & 63.55 & $F(3,254)=5.87$ & ** & **** & & \\
\hline & Very busy & 68.86 & 74.71 & 64.04 & 62.90 & $F(3,253)=5.25$ & ** & $* * *$ & & \\
\hline & Reliable & 66.00 & 72.21 & 55.61 & 54.52 & $F(3,253)=14.01$ & $* * *$ & $* * *$ & & \\
\hline & Dependable & 65.86 & 70.14 & 56.21 & 50.97 & $F(3,256)=13.91$ & $* * *$ & $* * *$ & & * \\
\hline & Reassuring & 64.86 & 68.57 & 54.92 & 53.33 & $F(3,255)=10.05$ & $* * *$ & $* * *$ & & \\
\hline & Trustworthy & 63.14 & 68.12 & 56.78 & 50.83 & $F(3,254)=10.55$ & $* * *$ & $* * *$ & & $*$ \\
\hline Fathers & Sociable & 60.56 & 66.38 & 57.93 & 56.13 & $F(3,257)=4.91$ & $* *$ & $* *$ & & $\dagger$ \\
\hline$\fallingdotseq$ & Robust & 60.00 & 65.94 & 55.09 & 50.81 & $F(3,255)=6.90$ & $* * *$ & $* * *$ & & * \\
\hline Mothers & Tough & 63.10 & 64.20 & 60.70 & 50.32 & $F(3,255)=5.81$ & $* *$ & $* *$ & $\dagger$ & $*$ \\
\hline & Brave & 60.42 & 63.19 & 50.00 & 46.94 & $F(3,256)=10.38$ & $* * *$ & *** & & \\
\hline & Cool & 50.74 & 57.50 & 47.54 & 37.54 & $F(3,250)=10.64$ & $* * *$ & $* * *$ & & ** \\
\hline & Courageous & 58.75 & 59.71 & 51.69 & 44.26 & $F(3,257)=8.98$ & $* * *$ & $* * *$ & & $\dagger$ \\
\hline & Polite & 63.24 & 63.57 & 54.07 & 61.11 & $F(3,259)=3.60$ & $*$ & $*$ & & \\
\hline & Sincere & 60.71 & 60.88 & 52.54 & 53.23 & $F(3,255)=4.20$ & ** & $* * *$ & & \\
\hline & Strict & 55.56 & 59.58 & 49.30 & 52.79 & $F(3,257)=3.71$ & $*$ & $* *$ & $\dagger$ & \\
\hline & Caring & 62.54 & 73.68 & 47.72 & 62.13 & $F(3,253)=20.10$ & $* * *$ & *** & **** & \\
\hline & Mature & 69.72 & 72.86 & 45.76 & 61.45 & $F(3,259)=24.87$ & $* * *$ & $* * *$ & $* * *$ & ** \\
\hline & Thoughtful & 61.74 & 71.74 & 56.03 & 60.49 & $F(3,253)=9.79$ & $* * *$ & $* * *$ & $* *$ & \\
\hline Fathers & Amazing & 59.58 & 71.18 & 47.72 & 56.77 & $F(3,254)=13.76$ & $* * *$ & $* * *$ & $* * *$ & \\
\hline$<$ & Kind & 64.37 & 70.29 & 59.48 & 62.42 & $F(3,256)=4.24$ & $* *$ & $* *$ & $*$ & \\
\hline Mothers & Watch over & 64.79 & 68.55 & 50.17 & 58.52 & $F(3,256)=11.37$ & $* * *$ & $* * *$ & $*$ & \\
\hline & Understanding & 58.47 & 68.55 & 45.00 & 55.97 & $F(3,257)=16.60$ & $* * *$ & $* * *$ & $* * *$ & \\
\hline & Fine & 62.82 & 69.30 & 52.03 & 55.16 & $F(3,259)=10.69$ & $* * *$ & $* * *$ & $*$ & \\
\hline & Generous & 56.03 & 64.93 & 49.48 & 51.61 & $F(3,251)=9.03$ & $* * *$ & $* * *$ & * & \\
\hline & Childrearing & 40.70 & 79.57 & 36.32 & 71.94 & $F(3,255)=87.15$ & *** & $*$ & *** & \\
\hline & Love family & 66.23 & 77.71 & 59.83 & 69.03 & $F(3,256)=11.66$ & $* * *$ & ** & $* * *$ & \\
\hline & Love children & 63.57 & 77.71 & 57.07 & 65.48 & $F(3,256)=13.93$ & $* * *$ & *** & *** & \\
\hline & Take children's sides & 60.87 & 77.50 & 57.97 & 67.42 & $F(3,254)=12.21$ & $* * *$ & $* *$ & *** & \\
\hline & Cherish family & 65.86 & 76.06 & 56.10 & 66.45 & $F(3,258)=12.51$ & $* * *$ & *** & *** & \\
\hline & Family oriented & 51.29 & 75.36 & 47.93 & 63.06 & $F(3,255)=27.75$ & $* * *$ & $* *$ & $* * *$ & $\dagger$ \\
\hline Miothers & Warm & 59.72 & 72.90 & 52.76 & 62.26 & $F(3,256)=15.27$ & *** & *** & *** & \\
\hline & Capacious & 54.71 & 71.86 & 53.79 & 58.52 & $F(3,255)=13.04$ & $* * *$ & $* *$ & $* * *$ & ** \\
\hline & Like children & 60.28 & 70.58 & 49.32 & 63.39 & $F(3,257)=16.38$ & $* * *$ & $* * *$ & $* * *$ & \\
\hline & Friendly & 54.29 & 68.55 & 48.10 & 56.45 & $F(3,255)=14.61$ & $* * *$ & $* * *$ & $* * *$ & \\
\hline & Interfering & 45.14 & 68.29 & 31.86 & 52.42 & $F(3,257)=47.19$ & $* * *$ & *** & *** & \\
\hline & Dedicated & 54.51 & 66.14 & 50.34 & 60.82 & $F(3,256)=9.76$ & $* * *$ & $*$ & *** & \\
\hline & Worrier & 52.36 & 65.88 & 47.89 & 58.23 & $F(3,255)=11.25$ & $* * *$ & $* *$ & $* * *$ & \\
\hline & Stabilize family & 60.00 & 63.86 & 52.07 & 62.90 & $F(3,255)=5.50$ & ** & $*$ & $* *$ & \\
\hline & Do housework & 38.87 & 76.62 & 36.21 & 70.16 & $F(3,255)=69.43$ & *** & $\dagger$ & *** & \\
\hline & Busy with childrearing & 27.46 & 73.82 & 27.76 & 70.00 & $F(3,255)=107.46$ & $* * *$ & & $* * *$ & \\
\hline & Cooperative & 54.64 & 64.93 & 54.83 & 62.26 & $F(3,254)=6.18$ & $* * *$ & & $* * *$ & \\
\hline & Emotional & 49.71 & 64.43 & 47.54 & 62.74 & $F(3,253)=12.68$ & $* * *$ & & $* * *$ & \\
\hline & Cheerful & 53.52 & 61.88 & 56.44 & 61.29 & $F(3,257)=4.61$ & ** & & $* *$ & \\
\hline & Preciuos & 34.20 & 58.12 & 35.09 & 54.84 & $F(3,253)=23.59$ & $* * *$ & & $* * *$ & \\
\hline & Pure & 41.55 & 52.43 & 36.21 & 51.13 & $F(3,257)=10.06$ & $* * *$ & & $* * *$ & \\
\hline & Soft & 37.36 & 61.88 & 37.37 & 66.72 & $F(3,255)=44.16$ & $* * *$ & & $* * *$ & \\
\hline$\fallingdotseq$ & Gracious & 50.14 & 57.50 & 47.02 & 63.06 & $F(3,254)=10.57$ & $* * *$ & & $* * *$ & $*$ \\
\hline & Calculating & 44.57 & 53.48 & 42.76 & 58.23 & $F(3,255)=9.78$ & $* * *$ & & $* * *$ & \\
\hline & Pretty & 32.82 & 54.00 & 35.52 & 57.54 & $F(3,256)=30.25$ & $* * *$ & & $* * *$ & \\
\hline & Sweet & 31.86 & 52.54 & 33.22 & 56.89 & $F(3,257)=26.12$ & $* * *$ & & $* * *$ & \\
\hline & Refined & 35.07 & 50.74 & 32.54 & 55.97 & $F(3,254)=25.44$ & $* * *$ & & $* * *$ & $\dagger$ \\
\hline & Tidy & 30.42 & 53.19 & 36.90 & 55.57 & $F(3,256)=24.56$ & $* * *$ & $\dagger$ & $* * *$ & \\
\hline & Elegant & 26.09 & 48.29 & 27.59 & 54.52 & $F(3,255)=35.19$ & $* * *$ & & $* * *$ & \\
\hline & Modest & 33.86 & 46.87 & 28.95 & 52.26 & $F(3,252)=20.58$ & $* * *$ & & $* * *$ & $*$ \\
\hline & Feminine & 26.48 & 53.94 & 35.25 & 67.21 & $F(3,258)=54.22$ & *** & *** & $* * *$ & \\
\hline & Pretty hair & 30.14 & 49.00 & 35.93 & 63.55 & $F(3,258)=37.80$ & $* * *$ & $* * *$ & $* * *$ & $\dagger$ \\
\hline & Feel lonely & 48.33 & 51.74 & 50.69 & 62.58 & $F(3,257)=6.67$ & $* * *$ & $* *$ & $* *$ & $\dagger$ \\
\hline Mothers & Blackhearted & 32.11 & 43.77 & 42.98 & 61.29 & $F(3,255)=23.62$ & $* * *$ & $* * *$ & $* * *$ & \\
\hline & Cute & 27.00 & 48.94 & 33.86 & 59.19 & $F(3,251)=32.79$ & $* * *$ & $* *$ & $* * *$ & \\
\hline & Brilliant & 26.34 & 46.97 & 32.41 & 57.90 & $F(3,253)=40.05$ & $* * *$ & $* * *$ & $* * *$ & \\
\hline & Beautiful & 23.53 & 49.13 & 30.68 & 56.39 & $F(3,253)=46.74$ & $* * *$ & $* *$ & $* * *$ & \\
\hline & Fresh & 37.68 & 52.90 & 46.90 & 52.90 & $F(3,254)=12.05$ & $* * *$ & $*$ & $* * *$ & $*$ \\
\hline Women & Coward & 32.32 & 46.67 & 47.07 & 59.02 & $F(3,253)=22.91$ & *** & $* * *$ & $* * *$ & \\
\hline$>$ Men & Mentally weak & 36.29 & 42.75 & 49.31 & 53.44 & $F(3,254)=9.51$ & $* * *$ & $* * *$ & $*$ & \\
\hline & Active & 50.74 & 58.70 & 57.89 & 55.32 & $F(3,252)=2.85$ & * & & & * \\
\hline & Moody & 46.44 & 49.85 & 53.73 & 53.55 & $F(3,257)=2.13$ & $\dagger$ & * & & \\
\hline & Quiet & 48.00 & 40.59 & 42.46 & 46.13 & $F(3,253)=2.44$ & $\dagger$ & & & $*$ \\
\hline Others & Immovable & 55.92 & 55.22 & 49.82 & 52.26 & $F(3,255)=1.65$ & & $*$ & & \\
\hline & Calm & 47.50 & 42.75 & 42.37 & 46.13 & $F(3,258)=1.54$ & & & & $*$ \\
\hline & Audacious & 50.71 & 55.57 & 53.39 & 48.69 & $F(3,256)=1.58$ & & & & $*$ \\
\hline & Stubborn & 55.63 & 55.57 & 54.48 & 51.13 & $F(3,257)=0.81$ & & & & \\
\hline
\end{tabular}


The mean age of respondents was $19.05(S D=1.02)$ years old. The respondents comprised 106 men $(39.85 \%)$ and 160 women (60.15\%). 73 (33 men and 40 women) answered the questionnaires on fathers, 71 ( 25 men and 46 women) answered those on mothers, 59 (20 men and 39 women) answered those on men and 63 ( 28 men and 35 women) answered those on women.

\subsection{Analysis of variance}

The data were analysed using a two-way analysis of variance. The analysis design used two independent variables: whether the target was parents or not (a between factor), and men or women (a between factor), and the dependent variable was each descriptor. Table 1 shows the results of analysis of variance.

First, it was found that the content of stereotypes of fathers, mothers, men, and women differed from each other. The respondents believed that fathers rather than men, mothers, and women have the seven characteristics Work, Hardworking, Breadwinner, Big, Masculine, Looking down on, and Well built.

They thought that mothers rather than women, fathers and men have the 14 characteristics: Childrearing, Love family, Love children, Take children's sides, Cherish family, Family oriented, Warm, Capacious, Like children, Friendly, Interfering, Dedicated, Worrier, and Stabilise family and that mothers rather than fathers, women and men have the 9 characteristics Caring, Mature, Thoughtful, Amazing, Kind, Watch over, Understanding, Fine, and Generous.

They believed that men rather than fathers, women, and mothers have the four characteristics Strong, Powerful, Lazy and Selfish. They thought that women rather than mothers, men, and fathers have the eight characteristics Feminine, Pretty hair, Feel lonely, Black hearted, Cute, Brilliant, Beautiful, and Fresh and that women rather than men, mothers and fathers have the two characteristics Coward and Mentally weak.

Next, it was also indicated that the content of parent stereotypes was different from that of overall gender stereotypes. The respondents believed that fathers and mothers rather than men and women have the following 15 characteristics: Busy, Very busy, Reliable, Dependable, Reassuring, Trustworthy, Sociable, Robust, Tough, Brave, Cool, Courageous, Polite, Sincere, and Strict.

On the other hand, the respondents thought that men and fathers rather than women and mothers have the 3 characteristics Physically strong, Strong constitution, and Work than children and mothers and women rather than fathers and men have the following 16 characteristics Do housework, Busy with childrearing, Cooperative, Emotional, Cheerful, Precious, Pure, Soft, Gracious, Calculating, Pretty, Sweet, Refined, Tidy, Elegant, and Modest. This means that the content of parent stereotypes overlaps with that of overall gender stereotypes.

\section{Discussion}

These results showed that the content of father and mother stereotypes differed from that of overall gender stereotypes and suggest that Japanese have the idea that 'fathers (rather than men) should work outside the home, and mothers (rather than women) should keep the house'. However, it should be noted that the content that repeated in parent stereotypes and overall gender stereotypes implies that 'both men and fathers should work outside the home, and both mothers and women should keep the house' and this point needs further verification.

Furthermore, Park, Smith, and Correll (2010) investigated not explicit but implicit stereotypes and found that fathers were strongly associated with professional images and mothers were more strongly associated with childcare images. Therefore, it will deepen our understanding to compare implicit parent stereotypes to implicit overall gender stereotypes. In addition, although this study targeted only undergraduates, future research should target older and/or less educated adults in order to generalise the findings.

\section{Acknowledgements}

This work was supported by JSPS KAKENHI Grant Number JP18K18298.

\section{References}

Ganong, L. H. \& Coleman, M. (1995). The content of mother stereotypes. Sex Roles, 32, 495-512.

Park, B., Smith, J. A., \& Correll, J. (2010). The persistence of implicit behavioural associations for moms and dads. Journal of Experimental Social Psychology, 46, 809-815.

Suzuki, A. (2017). Mechanisms of persistence in gender inequality: Perspective of work and family. Japanese Psychological Review, 60, 62-80.

Troilo, J. (2013). The drinker and the nurturer: College students' perceptions of men and fathers. Journal of Applied Social Psychology, 43, 1089-1096. 